\title{
Dicyclomine Hydrochloride
}

National Cancer Institute

\section{Source}

National Cancer Institute. Dicyclomine Hydrochloride. NCI Thesaurus. Code C28987.

The hydrochloride salt form of dicyclomine, a synthetic analog of acetylcholine with antimuscarinic activity. Dicyclomine hydrochloride antagonizes muscarinic receptors on smooth muscle in the gastrointestinal (GI) tract, thereby preventing the actions of acetylcholine and reducing Gl smooth muscle spasms. 